\title{
Evaluating Information-Centric Networks in Disconnected, Intermittent, and Low-Bandwidth Environments
}

\author{
Thiago Teixeira \\ University of Massachusetts Amherst \\ 151 Holdsworth Way \\ Amherst, Massachusetts \\ tteixeira@umass.edu
}

\author{
Michael Zink \\ University of Massachusetts Amherst \\ 151 Holdsworth Way \\ Amherst, Massachusetts \\ mzink@cas.umass.edu
}

\begin{abstract}
This paper studies information dissemination in wireless ad hoc networks, using standard routing protocols, such as OLSR, as well as Information-Centric Networking. We performed simulations using NS-3 and ndnSIM with different node counts and transport protocols. Our simulations show that TCP performs better in lower hop count scenarios, while NDN performs better in higher hop count scenarios.
\end{abstract}

\section{Keywords}

Information-Centric Networks; Mobile Ad hoc Networks

\section{INTRODUCTION}

In Mobile Ad hoc Networks (MANETs), nodes frequently experience disruptions and intermittent connectivity due to the inherent nature of the network, as nodes move in and out of communication range. Resource limitation caused by, e.g., battery operated radios with limited transmission power result in nodes transmitting with lower bandwidth to achieve a higher communication range. Mobile networks of such characteristics are called Disconnected, Intermittent, Low-bandwidth (DIL), in which the dissemination of information from sender to destination is quite challenging.

Existing routing protocols, such as Optimized Link-State Routing (OLSR) [1], aim to reduce the flooding of message in MANETs by selecting specific nodes, called multipoint relays (MPRs), to re-broadcast data packets, reducing collisions in the wireless channel. However, when nodes go out of communication range, thus, disrupting the end-to-end paths, intermediate nodes should take a store and forward approach in an attempt to push the content close to the user.

In a new approach, called Information-Centric Networking (ICNs) [4], content is addressed by its name, and not an IP address. The communication is started by the consumer by sending out an Interest packet, indicating the desired name of the content. The Interest packet is relayed until reaching the content source or a router with a cached version of the data. The data chunks then follow the breadcrumbs back

Permission to make digital or hard copies of part or all of this work for personal or classroom use is granted without fee provided that copies are not made or distributed for profit or commercial advantage and that copies bear this notice and the full citation on the first page. Copyrights for third-party components of this work must be honored. For all other uses, contact the owner/author(s).

ANCS '16 March 17-18, 2016, Santa Clara, CA, USA

(C) 2016 Copyright held by the owner/author(s).

ACM ISBN 978-1-4503-4183-7/16/03.

DOI: http://dx.doi.org/10.1145/2881025.2889483 to the consumer, leaving cached copies along the path. This dynamic in-network caching allows other nodes to retrieve the same content even when the source is no longer available.

Because of in-network caching, ICNs are specially interesting in ad hoc scenarios in which the network tends to partition. In this paper, we present a preliminary evaluation of a MANET routing protocol and ICN.

\section{METHOD}

In this work, we evaluate the performance of TCP and UDP using OLSR as routing protocol for wireless ad hoc networks. The evaluation is done via simulations using NS3 [6] and the Flow Monitor tool [3].

To simulate ICN, we use the Named-Data Networking simulator ndnSIM [5]. However, the current version of ndnSIM (ver. 2.1) does not support wireless multi-hop communication, as the Interest needs to be re-broadcast on the same face it was received from. Therefore, we use a modified forwarding strategy that allows nodes to re-broadcast Interests [2]. This strategy controls flooding with a timer-based suppression of re-broadcasts, without keeping information about its neighbor nodes. The basic idea is that nodes use a random defer timer to listen to the wireless channel, refraining from re-broadcasting packets if they overhear them. In our simulations, we explore different values of the defer timer.

\subsection{Simulation Scenario}

For simulating the scenarios, we used a line topology where each node in the network can only communicate with its immediate neighbors (i.e. node 3 sees only nodes 2 and 4 ). We varied the node count from two up to twelve nodes, with a few exceptions, as shown in Table 1. The consumer and producer are always on opposite ends of the topology. In one case, we deployed two consumers and two producers exchanging information at the same time to increase the channel utilization and analyze bi-directional traffic scenarios.

The random defer timer in NDN is controlled by the defer window size, as described in [2]. Figure 1 shows different window sizes, where in NDN-255 the nodes wait a longer time than NDN-63 before re-broadcasting the packets.

Each node is equipped with an $802.11 \mathrm{~b}$ interface, configured to $1 \mathrm{Mbps}$ to reflect a low-bandwidth scenario.

\section{RESULTS}

Figure 1 shows the average delay to retrieve one data packet for different node counts. We can split our analysis into two parts. First, looking at the left part of the 
Table 1: Simulation parameters

\begin{tabular}{cccc}
\hline Category & Parameter & TCP/UDP & ICN \\
\hline Application layer & Content store size & \multicolumn{2}{c}{1000 packets } \\
& Data payload & 1040 bytes \\
In-network caching & Content store size & 0 & 100 chunks \\
Wireless media & Propagation model & \multicolumn{2}{c}{ IEEE $802.11 \mathrm{~b}$ DSSS } \\
& Protocol & \multicolumn{2}{c}{$1 \mathrm{Mbps}$} \\
& Channel capacity & \multicolumn{2}{c}{$-80 \mathrm{dBm}$} \\
& Rx sensitivity & \multicolumn{2}{c}{$5 \mathrm{dBm}$} \\
Tx power & \multicolumn{2}{c}{$2,3,4,5,7,9$, and 12} \\
Node layout & Line topology & On/Off, Bulk 10 Interest per second \\
Consumer Application & Constant bit rate & TCP, UDP & NDN \\
Transport Layer & & N/A & 63,127, and 255 \\
NDN defer window size & &
\end{tabular}

graph, we notice that the fewer number of hops between the consumer and the producer, which leads to a fewer number of collisions, is not sufficient to produce a large number of retransmissions. This leads to a linear increase in delay as the node count increases. Thus, NDN does not benefit from caching, performing worse than its counterparts regardless of the defer window size. We attribute this to the fact that, for every data chunk, the consumer has to send an Interest request to the producer, therefore, having a higher overhead than TCP and UDP.

Second, for the right half of the graph ( $\geq 7$ nodes) the higher hop count leads to more traffic and, thus, collisions in the wireless channel, forcing the nodes to re-transmit packets more often. Due to the in-network caching of ICN lost data can potentially be retransmitted from any intermediate node, thus, making retransmissions more efficient, especially if the retransmitted data originate from caches close to the sink.

Since UDP is not a reliable protocol, we observe packet losses in the 7, 9, and 12 node cases, yielding a file completion rate of $75.9 \%, 82.6 \%$, and $70.4 \%$, respectively. For the TCP case, retransmissions starting to take a toll on the 7node case, making its performance worse than NDN-63 and NDN-127, and worse than NDN-255 after the 9-node case. This is due to the dynamic caching of the NDN protocol that allows more reliable communication, achieving $100 \%$ of file completion in all cases.

Furthermore, we simulated a bidirectional case where the end-point nodes are both consumers and producers, increasing the link utilization. This scenario aims to study the behavior of the aforementioned protocols in situations with higher collisions. As can be seen from NDN-127 Bidirectional, higher link utilization leads to a higher delay.

\section{CONCLUSIONS AND FUTURE WORK}

In this work, we investigated the use of information-centric networks in MANETs, drawing comparison with different transport and routing protocols. We used a well-known network simulator to evaluate different scenarios, where we observed that TCP performs better than NDN when the hop count is small. NDN, due to dynamic caching, is able to perform better than TCP when the hop count is higher. In future work, we plan to study mobility, different cache sizes, and more scenarios with multiple consumers and producers.

\section{ACKNOWLEDGMENTS}

The authors would like to thank Marica Amadeo from the Mediterranean University of Reggio Calabria for providing technical support for this work. The first author is supported by National Council for the Improvement of Higher

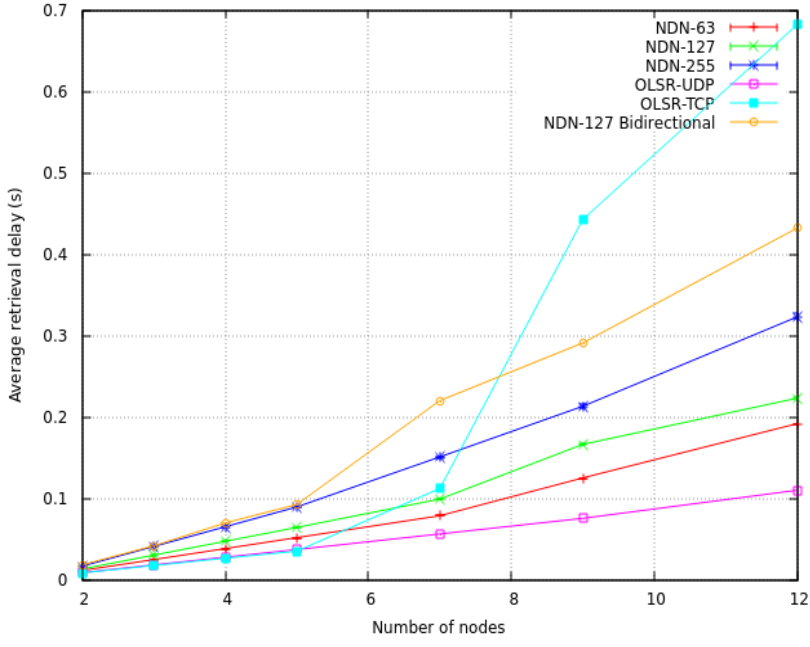

Figure 1: Average data retrieval delay per node for different protocols

Education (CAPES), Brazil. Partially funded by ONR contract number N00014-15-C-0122. Views presented are those of authors alone.

\section{REFERENCES}

[1] Optimized link state routing protocol (olsr), 2003.

[2] M. Amadeo, C. Campolo, and A. Molinaro. Forwarding strategies in named data wireless ad hoc networks: Design and evaluation. Journal of Network and Computer Applications, 50:148 - 158, 2015.

[3] G. Carneiro, M. Ricardo, and P. Fortuna. Flowmonitor - a network monitoring framework for the network simulator 3 (ns-3). In Proceedings of ICST NSTools 2009, Pisa, Italy, October 2009.

[4] V. Jacobson, D. K. Smetters, J. D. Thornton, M. F. Plass, N. H. Briggs, and R. L. Braynard. Networking named content. In Proceedings of the 5th International Conference on Emerging Networking Experiments and Technologies, CoNEXT '09, pages 1-12, New York, NY, USA, 2009. ACM.

[5] S. Mastorakis, A. Afanasyev, I. Moiseenko, and L. Zhang. ndnSIM 2.0: A new version of the NDN simulator for NS-3. Technical Report NDN-0028, NDN, January 2015.

[6] NS-3. https://www.nsnam.org. [Online; accessed 17-January-2016] 